J丁

INSTITUTE FOR

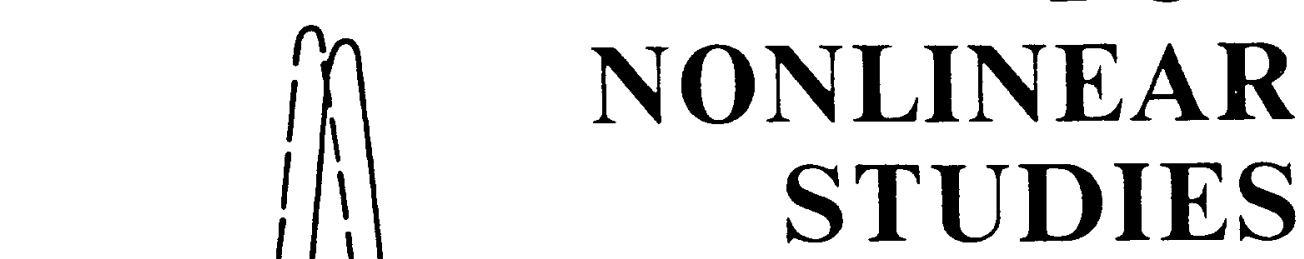

NONLINEAR EVOLUTION OF LANGMUIR AND ELECTROMAGNETIC PULSES

IN A WARM, UNMAGNETIZED PLASMA: MODUIATIONAL INSTABIIITY, INTEGRABILITY, AND SELF-FOCUSING IN $(2+1)$-DIMENSIONS

by

Ronald E. Kates* and D.J. Kaup December 1993

\section{CLARKSON UNIVERSITY}

Potsdam, New York 13699-5815, U.S.A.

* Astrophysical Institute Potscam

An der Sternwarte 16, 14482 Potsdam, GERMANY 


\begin{abstract}
The nonlinear dynamics of wave envelopes modulated in $(2+1)$ dimensions is considered for two systems in plasma physics: 1) Langmuir pulses and 2) intense (but weakly relativistic) electromagnetic (EM) pulses. Using singular perturbation techniques applied to an envelope approximation, both problems are reduced to the two-dimensional nonlinear Schrödinger system (2DNLS), which describes the dynamics of two coupled, slowly-varying potentials. The general 2 DNLS exhibits a rich variety of phenomena, including enhanced (compared to "longitudinal" propagation) modulational stability and (1D) soliton formation; decay of 1D solitons over long time scales; self-focusing regimes (determined by a virial type condition); as well as integrability and $2 \mathrm{D}$ solitons. Applying our recent results on the 2DNLS, we determine which of these phenomena can actually occur here and compute the parameter regimes: 1) The 2DNLS for the Zakharov equations is modulationally unstable for all parameter values. It also has an integrable sector and a self-focusing regime. 2) The 2DNLS describes coupled "longitudinal" and "transverse" modulations of linearly or circularly polarized EM pulses propagating through a warm, unmagnetized, two-component, neutral plasma with arbitrary masses (i.e., electron-positron or electronion). The pulse can accelerate particles to weakly (but not fully) relativistic velocities; relativistic, ponderomotive, and harmonic effects all contribute to the nonlinear terms. The resulting 2DNLS does not admit a self-focusing regime. Parameter values leading to an integrable case (the so-called "Davey-Stewartson I" equations, which admit 2D soliton solutions) are computed; however, the required values would not be attainable in a laboratory or astrophysical setting. Nonetheless, the existence of new nonlinear modulational instabilities associated with the second spatial degree of freedom already represents an important potential limitation on any $(1+1)$-dimensional approach to nonlinear evolution and modulational instability of plasma EM waves.
\end{abstract}




\section{Introduction and Model}

In a series of papers (Kates and Kaup 1989a,b; 1991; 1992; 1993 hereinafter Papers I-V) we have seen that problems involving the intense propagation of plane-fronted EM pulses are more subtle than they appear at first glance and require careful application of singular perturbation methods. The evolution of a modulated pulse is determined in general by the combined effects of linear dispersion, relativistic nonlinearity, nonlinear harmonic interactions, and ponderomotive nonlinearity. (Harmonic interactions vanish identically for circular polarization.) As a consequence, all of these effects may be significant in computing criteria for nonlinear modulational instabilities and possible soliton formation.

Unfortunately, (1+1)-dimensional ("1D") treatments of modulated EM pulses provide only part of the information needed for applications: In addition to modulation along the propagation direction, transverse modulations may be excited, i.e., the wave fronts may be slightly curved. This area of investigation has taken on added importance recently in view of recent experiments involving "channeled propagation" (Borisov, et. al, 1992a).

In those systems for which plane-fronted pulses satisfy the scalar 1D nonlinear Schrödinger equation (NLS)

$$
i q_{t}+q_{x x} \pm|q|^{2} q=0
$$

(see Papers I, II, and V), one might have supposed that generalization from 1D to 2D modulations of EM waves could have been accomplished simply by including transverse dispersion, i.e., by replacing the 1D dispersion term $q_{x x}$ in the NLS (1) with a $2 \mathrm{D}$ dispersion term:

where

$$
i q_{t}+\left[D_{x x} \partial_{x}^{2}+2 D_{x y} \partial_{x} \partial_{y}+D_{y y} \partial_{y}^{2}\right] q \pm|q|^{2} q=0
$$

$$
D_{i j} \equiv \frac{\partial^{2} \omega}{\partial k_{i} \partial k_{j}}
$$

A system of the form (2) is in fact obtained for a circularly polarized beam with modulations in both transverse directions but vanishing longitudinal modulations (Spatschek, 1977). This case has been called the "thin-beam" approximation.

Generally speaking, however, a proper description of 2D modulations of EM pulses involves two independent, coupled potential functions. This point was recognized by Karpman (1990). In particular, as we shall shortly see, Eqs. (2) will not provide the correct 2D evolution equation for a warm plasma if both longitudinal and transverse modulations are present, because fluid modes associated with longitudinal and transverse ponderomotive forces are coupled.

With this motivation, we recently studied (Kates \& Kaup, 1993b; hereinafter "Paper 0") a general class of two-dimensional nonlinear Schrödinger (2DNLS) systems, which can all be expressed in the "canonical" form

$$
i \partial_{t} q+O_{1} q=p q
$$




$$
\mathrm{O}_{2} p=\mathrm{O}_{3}\left(q^{*} q\right)
$$

where each of the operators $O_{n}$ in (3) is a second-order linear, symmetric (dispersion) operator of the form

$$
O_{n}=D_{x x}^{(n)} \partial_{x}^{2}+2 D_{x y}^{(n)} \partial_{x} \partial_{y}+D_{y y}^{(n)} \partial_{y}^{2}
$$

(Equations from Paper 0 will be prefaced by a " 0 ".) The 2DNLS (3) subsumes many special cases of interest, as described in Paper 0. These include the "DR" system (Djordjevic \& Redekopp, 1977; Ablowitz \& Segur, 1979), first studied in the context of water waves, and of considerable interest in its own right:

$$
\begin{gathered}
i A_{\tau}+\lambda A_{\xi \xi}+\mu A_{\eta \eta}=\chi A^{*} A^{2}+\chi_{1} A \Phi_{\xi} \\
\alpha \Phi_{\xi \xi}+\Phi_{\eta \eta}=-\beta\left(A^{*} A\right)_{\xi} .
\end{gathered}
$$

Any (1+1)-dimensional restriction of (3) (or (4)) satisfies (1).

The 2DNLS exhibits a rich variety of phenomena in various regimes, including enhanced modulational instability, self-focusing, integrability, and $2 \mathrm{D}$ soliton formation. The regimes of self-focusing and modulational stability are mutually exclusive, as are the conditions for integrability and self-focusing.

As shown in Paper 0 [Eq. (0.30)], the 2DNLS is modulationally stable (for given operators $\left.O_{n}, n=1,2,3\right)$ provided

$$
\mathcal{D}^{(1)} \mathcal{D}^{(2)} \mathcal{D}^{(3)}>0 \quad \forall \vec{K}
$$

where $\vec{K}$ is the wavenumber of the modulation, and where

$$
\mathcal{D}^{(n)}=K_{i} K_{j} D_{i j}^{(n)}
$$

See Eq. (3) for the definition of the $D_{i j}^{(n)}$ in terms of the $O_{n}$.

If $(5 a)$ is not satisfied for some $\vec{K}$, then the system is modulationally unstable to modulations in the direction $\hat{K}$ (see Paper 0) for sufficiently small $K(=|\vec{K}|)$. The 1D projection (defined by assuming spatial dependence on $y=\hat{K} \cdot \vec{x}$ ) satisfies the NLS (1) with the upper (modulationally unstable) sign and thus admits $1 D$ soliton solutions.

The self-focusing regime of (3) is characterized by the eigenvalues of the $D_{i j}^{(n)}: O_{1}$ and $\mathrm{O}_{2}$ must both be elliptic, and for that choice of variables for which $D_{i j}^{(1)}$ and $D_{i j}^{(2)}$ both have positive signature, $D_{i j}^{(3)}$ must have at least one negative eigenvalue.

The integrable cases of DR, which are known as the Davey-Stewartson equations (DS-I and DS-II), as well as all other integrable cases of (3), may be obtained from the following conditions: First,

at least one of $\mathrm{O}_{2}$ and $\mathrm{O}_{3}$ must not be elliptic; 
second,

$$
D_{i j}^{(1)}=\kappa D_{i j}^{(3)}
$$

must be satisfied for some constant $\kappa$; third,

$$
\left.\sum_{i j} D_{i j}^{(2)}\left[\left(D^{(3)}\right)^{-1}\right]_{j i}\right)=0
$$

and fourth

$$
\operatorname{det}\left(D^{(n)}\right) \neq 0 \text { for each of } n=1,2,3 .
$$

The cases admitting $2 \mathrm{D}$ solitons were discussed in Paper 0.

Consider a system that reduces to the (1D) NLS (1) in some 1D subspace (e.g., modulations in the $\hat{z}$ direction). If this $1 \mathrm{D}$ reduction is modulationally stable, the system may still be unstable with respect to modulations in a different direction. In particular, it may self-focus or, if it happens to be integrable, form 2D solitons. Similarly, if a 1D reduction is modulationally unstable and forms $1 \mathrm{D}$ solitons, these $1 \mathrm{D}$ solitons may decay due to transverse perturbations. These properties of (3) pose a potential limitation on the validity of conclusions drawn from a $1 \mathrm{D}$ analysis of any intrinsically multi-dimensional, NLS-type system.

In Section 2, we show that the canonical form (3) of the 2DNLS describes the Zakharov equations (Zakharov, 1972) in an envelope approximation. This computation illustrates the methods used in Section 3, and it provides an example of a nontrivial 2DNLS system in canonical form (3) that does not always reduce to the DR form (4). In this approximation, the Zakharov equations are always modulationally unstable. They contain an integrable case as well as a self-focusing regime.

In Paper I we considered 1D modulations of a plane-fronted pulse with arbitrary polarization, propagating in an unmagnetized (possible warm) plasma. In Section 3, we consider the same model for the plasma but allow 2D modulations. As in Papers I, II, and $\mathrm{V}, \epsilon \equiv \frac{e}{m c \omega}|E|$ is a dimensionless measure, both of the typical velocity of electrons excited by the wave, and of the slowness of the modulations, but here the "modulations" depend on both $y$ and $z$, where $\hat{z}$ is the propagation direction. We give the explicit computation for linear polarization along $\hat{x}$ (circular polarization may be treated similarly). Consequences are discussed in Sect. 4: In the present case of an unmagnetized plasma, it turns out that the effects of finite temperature are crucial in determining the qualitative behavior. In particular, for an electron-positron plasma the frequency range for modulational instability including both transverse and longitudinal modes is considerably broader than it was for purely longitudinal modulations (Paper I).

We emphasize that in a self-consistent approximation procedure, the effects of linear dispersion, relativistic nonlinearity, nonlinear harmonic interactions, and ponderomotive nonlinearity will generally enter at the same order with respect to $\epsilon$, just as in the $(1+1)$ dimensional case. In the treatment of Karpman and Washimi (1977), relativistic effects were not included (see Shukla \& Stenflo, 1984). In the treatment of Borisov et al (1992b), 
the approximation $\vec{p}_{e}=\vec{A}$ stated below their Eq. (8) takes into account relativistic corrections (compare our Eqs. (5) below) but not pondermotive forces (see Eqs. (32) - (42) below or (I.20) in the (1+1)-dimensional case). If one is interested in electron-positron plasmas, ponderomotive effects are at least as large as the other nonlinear effects and must always be included. Now, in $1 \mathrm{D}$ systems, nonlinear ponderomotive effects in an ion-electron plasma are usually down by a factor of $m / M$ compared to relativistic and harmonic effects. However, in the coefficient $\beta$ in Eq. (103b), which gives the strength of the coupling to the potential field $\Phi$, (transverse) ponderomotive forces contribute to the leading terms. Whether or not this coupling has a short enough timescale to be qualitatively important will of course depend on the details of the particular physical situation.

\section{Reduction of the Zakharov equations to the 2DNLS}

The Zakharov equations (Zakharov, 1972) arose in models of the multidimensional interaction of acoustic waves with an electrostatic (Langmuir) plasma wave. After normalization, the Zakharov equations for one linearly polarized mode may written as

$$
\begin{gathered}
\partial_{t}^{2} P-c_{s}^{2} \nabla^{2} P=\nabla^{2}\left(Q^{*} Q\right) \\
i \partial_{t} Q+\nabla^{2} Q=P Q
\end{gathered}
$$

where $c_{s}$ is proportional to the sonic velocity, and damping is not considered. Eqs. (7) exhibit collapse and other phenomena associated with nonintegrable equations. If there exists a limit for which the $\partial_{t}^{2} P$ term may simply be neglected, (7) evidently reduces to a pure (multidimensional) nonlinear Schrödinger equation (2). However, as shown in Paper 0 , for integrability an auxiliary potential must be present. This may be achieved by a different limiting procedure:

One expands $P$ and $Q$ in terms of a small parameter $\epsilon$, taking the leading term $Q_{1}$ in $Q$ to be of first order and the leading term $P_{2}$ in $P$ to be of second order in $\epsilon$. One then introduces slow coordinates

$$
X \equiv \epsilon x, \quad Y \equiv \epsilon y \quad T \equiv \epsilon t, \quad \tau \equiv \epsilon^{2} t
$$

and assumes an envelope approximation for $Q_{1}$ of the form

$$
Q_{1}=a e^{i(k x-\omega t)}
$$

(For convenience, the wave vector is chosen without loss of generality to be in the $x$ direction. There is still slow dependence in both spatial directions.) Both $P_{2}$ and a are assumed to.depend only on the slow variables. The first-order terms yield a dispersion relation

$$
\omega=k^{2}
$$

while elimination of secular terms at second order leads to the group velocity condition

$$
\left(\partial_{T}+v_{g} \partial_{X}\right) a=0
$$


where

$$
v_{g}=\frac{d \omega}{d k}=2 k
$$

The third-order secular condition on (7b) yields

$$
i a_{\tau}+a_{X X}+a_{Y Y}=P_{2} a,
$$

while the leading (third-order) term in $(7 \mathrm{a})$ is

$$
v_{g}^{2} \partial_{X}^{2} P_{2}-c_{s}^{2}\left(\partial_{X}^{2}+\partial_{Y}^{2}\right) P_{2}=\left(\partial_{X}^{2}+\partial_{Y}^{2}\right) a^{*} a
$$

Eqs. (12) are of the canonical 2DNLS form (3), with

$$
\begin{gathered}
O_{1}=\partial_{X}^{2}+\partial_{Y}^{2} \\
O_{2}=\left(v_{g}^{2}-c_{s}^{2}\right) \partial_{X}^{2}-c_{s}^{2} \partial_{Y}^{2} \\
O_{3}=\partial_{X}^{2}+\partial_{Y}^{2}=O_{1}
\end{gathered}
$$

The integrability conditions (6) then reduce to the requirement

$$
v_{g}^{2}=4 k^{4}=2 c_{s}^{2},
$$

which gives the DS-I form. Thus we see that a sector exists for which the Zakharov equations are integrable in $(2+1)$ dimensions: namely, where the Langmuir wave is a small amplitude, almost plane wave with the group velocity near $\sqrt{2} c_{s}$. Incidentally, this computation illustrates the utility of expressing integrability conditions for the 2DNLS in a coordinate-free, canonical form: this integrable sector of the Zakharov equations appears to have been unknown until now.

We can also use the canonical 2DNLS form (3) to study modulational instability by using (13) - (15) in (5): Since both $\mathcal{D}^{(1)}$ and $\mathcal{D}^{(3)}$ are positive definite, modulational stability requires

$$
\mathcal{D}^{(2)}>0
$$

which in view of (14) is always violated for some $\vec{K}$. This result appears to be consistent with numerical investigations (Goldman, 1984). if

Eqs. (12) satisfy the self-focusing conditions of Paper 0, which are of the "virial" type,

$$
v_{g}^{2}=4 k^{4}<c_{s}^{2}
$$

This result may also shed some light on observations and would seem to be consistent with the existence of self-similar solutions of the full equations exhibiting collapse (Goldman, 1984). 


\section{Reduction of EM-pulse propagation in a warm plasma to the 2DNLS}

In light of the preceeding results, we now return to the problem considered in Paper I: the long-term evolution of a modulated pulse propagating in an unmagnetized (possible warm) plasma. For economy, we preface equations of Paper I by "I" and follow the notation of that paper unless otherwise stated. We take the $z$ axis in the direction of propagation $(\vec{k}=\hat{z} k)$ as in Paper I but permit slow modulations along both the $z$ and $y$ directions. In the following computation, we assume linear polarization in the $x$ direction.

The system (I.4-I.6) to be solved (Maxwell's equations and two sets of fluid equations) describes a fully-ionized plasma composed of singly-ionized atoms (of charge $+e$ and mass $M$ ) and electrons (of charge $-e$ and mass $m$ ); the plasma is modeled as a perfect fluid with constant but possibly distinct electron and ion temperatures. No approximation is made with respect to $m / M$ unless explicitly stated. (Upon setting $M=m$, we recover the electron-positron case.) The background plasma is taken to be unmagnetized and neutral. (There will of course be deviations from neutrality due to nonlinear effects.) Units are chosen such that $c=1$ unless otherwise stated.

Mathematically, the intended generalization of Paper I may be stated as follows: we study the evolution of appropriate initial data given in the plane $-\infty<z<\infty$, $-\infty<y<\infty$ for the functions $n, \vec{v}, N, \vec{V}, \vec{A}, \phi$ (the electron density and velocity, the ion density and velocity, the vector potential, and the electric potential, respectively). We are interested in solutions that satisfy the original equations up to appropriate order (as explained in Papers I-V) and that correspond to slowly modulated, almost plane waves. We assume that well-posed initial data can be given which evolve in this way. All results then follow from substitution of the expansions into the field equations and collection of terms order by order. Some of the equations are to be solved directly, while others yield integrability conditions.

Asymptotic expansions are assumed according to (I.7-I.8), where $n_{0}=$ const, $N_{0}=n_{0}$, and all other zeroth-order quantities vanish. The slow variables are

$$
Z \equiv \epsilon z, \quad Y \equiv \epsilon y \quad T \equiv \epsilon t, \quad \tau \equiv \epsilon^{2} t
$$

With this scaling, the effects of linear dispersion (with respect to both $z$ and $y$ ), relativistic corrections, harmonic interactions, and ponderomotive forces will enter at the same (third) order of our approximation scheme.

The linearized theory is given by (I.11 - I.13), with

$$
\overrightarrow{A_{1}}=\vec{a} e^{i(k z-\omega t)}+\text { c.c. }
$$

and $\vec{a}$ in the $x-y$ plane. Here, we assume

$$
\vec{a}=a \hat{x}
$$

As in Papers I-V, a will now be permitted to depend on the above slow coordinates. We collect like powers of $\epsilon$ in all equations and enforce the nonsecularity conditions of 
Paper I (see I.15-16). As usual, the first-order terms are identical to the linear theory. while slow derivatives in $a$ will enter the higher-order equations. Additional slowly varying quantities will be introduced as required. In particular, terms will be classified as "DC" $(0)$, "fundamental" (1), "harmonic" (2), etc., as in Papers I-V. For example, the second-order electron density $n_{2}$ is decomposed as

$$
n_{2}=n_{2}^{(0)}+\left[n_{2}^{(1)} e^{i(k z-\omega t)}+\text { c.c. }\right]+\left[n_{2}^{(2)} e^{2 i(k z-\omega t)}+\text { c.c. }\right]
$$

and the notation for other variables is analogous. Note that coefficients such as $n_{2}^{(0)}$ generally depend on the slow variables.

As before, we may take

$$
\begin{gathered}
n_{2}^{(1)}=N_{2}^{(1)}=\phi_{2}^{(1)}=0 \\
{\overrightarrow{v_{2}}}^{(1)}={\overrightarrow{v_{2}}}^{(1)}={\overrightarrow{A_{2}}}^{(1)}=0 .
\end{gathered}
$$

without loss of generality.

Elimination of secular terms at second order leads to the group velocity condition

$$
\left(\partial_{T}+v_{g} \partial_{Z}\right) a=0
$$

where as before

$$
v_{g}=\frac{d \omega}{d k}=k / \omega
$$

The absence of terms involving $\partial_{Y}$ is simply due to the definition of $z$ as the direction of propagation.

The harmonic terms at second order may be shown to be given by

$$
\begin{aligned}
& f_{2} n_{2}^{(2)}+F_{2} N_{2}^{(2)}=\frac{k^{2} \omega_{p}^{2}}{8 \pi} a^{2} \\
& \frac{n_{2}^{(2)}}{N_{2}^{(2)}}=\frac{4 m f_{2}-m M \omega_{p}^{2}}{4 M F_{2}-m M \omega_{p}^{2}} \\
& f_{2} \equiv m\left(\omega^{2}-c_{s}^{2} k^{2}\right) \\
& F_{2} \equiv M\left(\omega^{2}-C_{s}^{2} k^{2}\right) .
\end{aligned}
$$

These terms agree with those of those of Paper I (given for vanishing pressure by (I.21)). This is to be expected, since they depend only algebraically on $a$.

The first-order DC parts of the fluid equations imply, with the second-order DC part of Poisson's equation (I.6b),

$$
n_{2}^{(0)}=N_{2}^{(0)}
$$


and with the second-order DC part of Maxwell's equation (I.6c),

$$
\begin{aligned}
& \vec{V}_{2}^{(0)}=\vec{v}_{2}^{(0)} \\
& \vec{A}_{2}^{(0)}=0 .
\end{aligned}
$$

However, determination of the the second-order DC quantities requires evaluation of the third-order fluid equations and will be discussed shortly.

One can again take

$$
\phi_{3}^{(1)}=N_{3}^{(1)}=n_{3}^{(1)}=0, \quad \vec{A}_{3}^{(1)}=0
$$

without loss of generality. The relativistic corrections again produce third-order perturbation of the velocities of the form

$$
\begin{aligned}
& \vec{v}_{3}^{(1)}=\frac{-3 e^{3}}{2 m^{3} c^{5}} a^{2} a^{*} \hat{x} \\
& \vec{V}_{3}^{(1)}=\frac{+3 e^{3}}{2 M^{3} c^{5}} a^{2} a^{*} \hat{x} .
\end{aligned}
$$

(Note again that these derive from terms depending algebraically on $a$.)

¿From the fundamental part of the $z$-component of the $\vec{\nabla} \times \vec{B}$ equation at third order we obtain the secular condition

$$
\begin{aligned}
& 2 i \omega \partial_{\tau} a+\left(c^{2} \partial_{Z}^{2}+c^{2} \partial_{Y}^{2}-\partial_{T}^{2}\right) a-\omega_{p}^{2} a\left(n_{2}^{(0)} / n_{0}\right) \\
& -\omega_{p}^{2} a^{*}\left[\frac{n_{2}^{(2)}}{m n_{0}}+\frac{N_{2}^{(2)}}{M n_{0}}\right] \frac{1}{\frac{1}{m}+\frac{1}{M}}+\frac{3 e^{2} \omega_{p}^{2}}{2 c^{4}} \frac{\frac{1}{m^{3}}+\frac{1}{M^{3}}}{\frac{1}{m}+\frac{1}{M}} a^{*} a^{2}=0
\end{aligned}
$$

We now come to the second-order DC terms $\left(n_{2}^{(0)}, N_{2}^{(0)}\right.$, etc.): In contrast to Paper I, these may not all be expressed algebraically in terms of $a$ (except in the case of vanishing pressure). We first introduce the abbreviations

$$
\begin{aligned}
q & \equiv n_{2}^{(0)} / n_{0} \\
\vec{\nabla}_{1} & \equiv \hat{y} \partial_{Y}+\hat{z} \partial_{Z} .
\end{aligned}
$$

The third-order DC parts of (I.4b) and (I.5b) may be added to eliminate the second-order DC potential, yielding

$$
(m+M) \partial_{T} \vec{v}_{2}^{(0)}+e^{2}\left(\frac{1}{m}+\frac{1}{M}\right) \vec{\nabla}_{1}\left(a^{*} a\right)+\left(m c_{s}^{2}+M C_{s}^{2}\right) \vec{\nabla}_{1} q=0
$$

¿From the continuity equation (I.4a) at the same order, we have

$$
\partial_{T} q+\vec{\nabla}_{1} \cdot \vec{v}_{2}^{(0)}=0 .
$$


We now let

$$
\vec{v}_{2}^{(0)}=\left(\partial_{Z} \psi\right) \hat{y}+w \hat{z}
$$

Note that $\vec{v}_{2}^{(0)}$ now has both $y$ and $z$ components, in contrast to (I.20b). Like $a$, the quantities $q, \psi$, and $w$ are functions of the slow variables.

Since we are interested in solutions driven by $a$, we can eliminate $T$ derivatives using

$$
\begin{aligned}
& \partial_{T} a=-v_{g} \partial_{Z} a \\
& \partial_{T} q=-v_{g} \partial_{Z} q \\
& \partial_{T} w=-v_{g} \partial_{Z} w \\
& \partial_{T} \psi=-v_{g} \partial_{Z} \psi,
\end{aligned}
$$

where $(37 \mathrm{a})$ is just $(24 \mathrm{a})$ and $v_{g}$ is given in $(24 \mathrm{~b})$. It is convenient to define

$$
v_{s}^{2} \equiv \frac{m c_{s}^{2}+M C_{s}^{2}}{(m+M)}
$$

The $z$-component of (34) may then be integrated to obtain

$$
w=\frac{e^{2}}{m M v_{g}} a a^{*}+v_{s}^{2} q / v_{g},
$$

while integration of the continuity equation (35) yields

$$
q=\left(w+\partial_{Y} \psi\right) / v_{g}
$$

In each of the above integrations, we have assumed that the various quantities vanish at the front of the pulse and that the pulse is entering a quiescent region. Substitution of (39) into (40) then gives

$$
q=\frac{1}{v_{g}^{2}-v_{s}^{2}}\left(\frac{e^{2}}{m M}\left(a^{*} a\right)+v_{g} \partial_{Y} \psi\right)
$$

Inserting the $Z$-derivative of (37d) and the $Y$-derivative of (41) into the $y$-component of (34) then yields

$$
\left(v_{g}^{2}-v_{s}{ }^{2}\right) \psi_{Z Z}-v_{s}^{2} \psi_{Y Y}=\frac{e^{2} v_{g}}{m M}\left(a^{*} a\right)_{Y} .
$$

This is coupled to the secular condition (31), which may be rewritten as

$$
2 i \omega a_{\tau}+\frac{\omega_{p}^{2} c^{2}}{\omega^{2}} a_{Z Z}+c^{2} a_{Y Y}+\left(C_{H}+3 C_{R}+C_{P}\right) a^{*} a^{2}-\frac{\omega_{p}^{2} v_{g}}{v_{g}^{2}-v_{s}^{2}} \psi_{Y} a=0
$$

where, as derived in Paper I, $C_{H}, C_{R}$, and $C_{P}$ are given by 


$$
\begin{aligned}
C_{H}=-\frac{e^{2} k^{2} \omega_{p}^{2}}{2 c^{2}}\left(\frac{m M}{M+m}\right)\{ & \frac{1}{m^{3}\left(\omega^{2}-k^{2} c_{s}^{2}\right)}+\frac{1}{M^{3}\left(\omega^{2}-k^{2} C_{s}^{2}\right)} \\
& \left.+\frac{\omega_{p}^{2}}{4 D_{2}}\left(\frac{m M}{M+m}\right)\left[\frac{1}{m^{2}\left(\omega^{2}-c_{s}^{2} k^{2}\right)}-\frac{1}{M^{2}\left(\omega^{2}-C_{s}^{2} k^{2}\right)}\right]^{2}\right\}
\end{aligned}
$$

with

$$
D_{2} \equiv 1-\frac{1}{4} \omega_{p}^{2} \frac{m M}{M+m}\left[\frac{1}{m\left(\omega^{2}-c_{s}^{2} k^{2}\right)}+\frac{1}{M\left(\omega^{2}-C_{s}^{2} k^{2}\right)}\right]
$$

and

$$
\begin{gathered}
C_{R}=\frac{e^{2} \omega_{p}^{2}}{2 c^{4}} \frac{\frac{1}{m^{3}}+\frac{1}{M^{3}}}{\frac{1}{m}+\frac{1}{M}} \\
C_{P}=-\frac{e^{2}}{c^{2}} \omega^{2}\left(\frac{M+m}{M m}\right) \frac{1}{M\left(v_{g}^{2}-C_{s}^{2}\right)+m\left(v_{g}^{2}-c_{s}^{2}\right)}
\end{gathered}
$$

The system of equations (42)-(43) may be recognized as the DR form of the 2DNLS system (0.3) if we identify $\Phi=\psi, A=a, \xi=Y, \eta=Z$ and redefine $\tau$ of Eq. (0.3) as $\tau /(2 \omega)$. The constants appearing in (4) are then given by

$$
\begin{gathered}
\chi=-\left(C_{H}+3 C_{R}+C_{P}\right) \\
\beta=-\frac{e^{2} v_{g}}{m M\left(v_{g}^{2}-v_{s}^{2}\right)} . \\
\lambda=1 \\
\mu_{0}=\frac{\omega_{p}^{2}}{\omega^{2}} \\
\chi_{1}=\frac{\omega_{p}^{2} v_{g}}{v_{g}^{2}-v_{s}^{2}} \\
\alpha=-\frac{v_{s}^{2}}{v_{g}^{2}-v_{s}^{2}}
\end{gathered}
$$

If the computations of this section are repeated for the case of circular polarization, the system may again be reduced to the form (4), but Eqs. (47a) and (47b) are replaced by

$$
\begin{aligned}
& \chi=-2\left(C_{P}+2 C_{R}\right) \quad(\text { circular polarization }) \\
& \beta=-\frac{2 e^{2} v_{g}}{m M\left(v_{g}^{2}-v_{s}^{2}\right)} \quad \text { (circular polarization) }
\end{aligned}
$$


In terms of the canonical form (3), we have for both linear and circular polarization

$$
\begin{gathered}
O_{2}=\frac{v_{g}^{2}-v_{s}^{2}}{\omega_{p}^{2} v_{g}}\left(\partial_{Z}^{2}-\frac{v_{s}^{2}}{v_{g}^{2}-v_{s}^{2}} \partial_{Y}^{2}\right) \\
O_{3}=\frac{\chi\left(v_{g}^{2}-v_{s}^{2}\right)}{\omega_{p}^{2} v_{g}} \partial_{Z}^{2}-\left[\beta+\frac{\chi v_{s}^{2}}{\omega_{p}^{2} v_{g}}\right] \partial_{Y}^{2} \\
O_{1}=\frac{\omega_{p}^{2}}{\omega^{2}} \partial_{Z}^{2}+\partial_{Y}^{2}
\end{gathered}
$$

with $q=a$ and

$$
p=\chi a^{*} a+\frac{\omega_{p}^{2} v_{g}}{v_{g}^{2}-v_{s}^{2}} \psi_{Y},
$$

where $\chi$ and $\beta$ are as given in (47) for linear polarization and (47') for circular polarization.

\section{Consequences of 2DNLS for EM pulses}

Now that the problem of EM wave propagation as considered in Section 3 has been reduced to the 2DNLS (3), several interesting features of the system may be determined from the operators in (48).

\subsection{No self-focusing regime}

The coefficients (47) always lie outside the self-focusing regime defined in Paper 0: In terms of DR notation (4), one observes if $\alpha>0$, then $\beta$ and $\chi_{1}$ must have opposite signs. The computation of Section 3 thus predicts that violent self-focusing singularities of the kind discussed in Section 3 will not arise for 2D (longitudinal - transverse) modulations of linearly or circularly polarized, nearly plane EM waves in a warm, unmagnetized plasma.

\subsection{Integrability conditions}

Conditions for integrability of (3) were derived in Paper 0 and given in Eqs. (6). For the operators defined in (48), we first observe that $\mathrm{O}_{3}$ is always elliptic. From (6a), $\mathrm{O}_{2}$ must also be elliptic. Then Eq. (6b) can only be satisfied if $O_{1}$ is hyperbolic. Thus, the only possible integrable case is DS-I. Eqs. $(6 \mathrm{~b}, \mathrm{c})$ [or equivalently $(0.18)$ ] then imply

$$
\begin{aligned}
\frac{e^{2} v_{g}^{2} \omega_{p}^{4}}{m M \omega^{2}\left(v_{g}^{2}-v_{s}^{2}\right)^{2}} & =-2\left(C_{H}+3 C_{R}+C_{P}\right) \\
v_{s}^{2} \omega_{p}^{2} & =\omega^{2}\left(v_{g}^{2}-v_{s}^{2}\right)
\end{aligned}
$$


For the case of a positron-electron plasma, this would require unphysical values $v_{s}^{2}=3.74 c^{2}$ and $k^{2}$ negative. For an electron-positive ion plasma, conditions (139) can in fact be (formally) satisfied if $v_{s}^{2}=0.776 c^{2}$ (which is of course highly relativistic) and $k^{2} / \omega_{p}^{2}=6.92$. Thus, in either case, our theory predicts no 2D solitons for EM waves in an unmagnetized, warm plasma such as could be maintained in a laboratory. (The same may be shown for circular polarization.) Nevertheless, it is interesting from a theoretical point of view that there is no fundamental obstacle to obtaining DS-I from plasma electromagnetic waves.

\subsection{Broadened range for modulational instability}

In Paper I, we found that for an ion-electron plasma modulational instability already occurs for purely longitudinal propagation within a broad range of frequencies [see Eqs. (I.28)]. However, for an electron-positron plasma, the case of interest for pulsar micropulses, the corresponding frequency range was quite narrow. The most dramatic consequence of the 2DNLS in a warm, unmagnetized plasma is that the frequency range for modulational instability in an electron-positron plasma is also broad if transverse modulations are considered. This result may be derived in a straightforward manner:

Since from (48c) $O_{1}$ is positive definite, the requirement (5) for modulational stability in either an ion-electron plasma or an electron-positron plasma reduces to

$$
\left[\cos ^{2} \theta-\frac{v_{s}^{2}}{v_{g}^{2}-v_{s}^{2}} \sin ^{2} \theta\right] \cdot\left[\chi\left(\cos ^{2} \theta-\frac{v_{s}^{2}}{v_{g}^{2}-v_{s}^{2}} \sin ^{2} \theta\right)+\frac{e^{2} v_{g}^{2} \omega_{p}^{2}}{m M\left(v_{g}^{2}-v_{s}^{2}\right)^{2}} \sin ^{2} \theta\right]>0,
$$

where we have used $K_{z}=K \cos \theta, K_{y}=K \sin \theta, \vec{K}$ being again the wavevector of the modulation. [Eq. (51) may also be derived from the DR dispersion relation, Eq. (0.34b).]

Modulation just along the $z$-direction corresponds to setting $\theta=0$ in (51). In this case modulational stability depends purely on the sign of $\chi$ (positive $\chi \rightarrow$ stable) and reduces to the results of Paper I. However, positive $\chi$ does not ensure modulational stability for arbitrary $\theta$ : Suppose $\chi>0$ and $v_{g}>v_{s}>0$ (the usual case). We can always choose $\theta$ in some neighborhood of $\pi / 2$ such that

$$
\left[\cos ^{2} \theta-\frac{v_{s}^{2}}{v_{g}^{2}-v_{s}^{2}} \sin ^{2} \theta\right]<0
$$

while keeping the second factor in (51) positive. In this angular range, the system is modulationally unstable, and "transverse" $1 \mathrm{D}$ solitons are possible.

This "transverse" instability is of particular interest for a plasma consisting of positrons and electrons with equal, nonvanishing (but possibly low) speeds of sound $c_{ \pm}$. As shown in Paper I, for either linear or circular polarization, modulations along the propagation direction are modulationally stable $(\chi>0)$ except for a narrow range of frequencies above 
the plasma frequency: For linear polarization, this range may be determined from the condition $\chi<0$, with $\chi$ given by $(47 \mathrm{a})$ and

$$
C_{P}+3 C_{R}+C_{H}=\frac{e^{2} \omega_{p}^{2}}{m^{2} c^{4}}\left[\frac{1}{2}\left(\frac{\left(1+c_{ \pm}^{2} / c^{2}\right) \omega_{p}^{2}-c_{ \pm}^{2} / c^{2} \omega^{2}}{\left(1-c_{ \pm}^{2} / c^{2}\right) \omega^{2}+c_{ \pm}^{2} / c^{2} \omega_{p}^{2}}\right)-\left(\frac{\omega_{p}^{2}+c_{ \pm}^{2} / c^{2} \omega^{2}}{\omega^{2}\left(1-\frac{c_{ \pm}^{2}}{c^{2}}\right)-\omega_{p}^{2}}\right)\right]
$$

Similarly, the unstable frequency range for circular polarization follows from the condition $\chi<0$, with $\chi$ given by $\left(47 a^{\prime}\right)$ and

$$
C_{p}+2 C_{R}=\frac{e^{2} \omega_{p}^{2}}{m^{2} c^{4}}\left(1-\frac{c^{2}}{v_{g}^{2}-c_{ \pm}^{2}}\right)
$$

(Note that Eq. (I.29c) contains a misprint: in the numerator of the fraction, $\omega_{p}^{4}$ should appear instead of $\omega_{p}^{2}$. The speed of light appears explicitly in (53) but is otherwise taken to be unity.)

Summarizing, if modulations at an arbitrary angle $\theta$ are permitted, the preceeding discussion shows that modulational instability in a warm positron-electron plasma becomes possible within a broad range of frequencies. For fixed $v_{s}$, the range of angles $\theta$ about $\pi / 2$ is usually small, and it approaches zero for fixed $\omega$ as $v_{s} \rightarrow 0$. The angular range increases (for fixed, nonzero $v_{s}$ ) as $\omega$ decreases toward the plasma frequency until it includes the longitudinal direction.

\subsection{Physical interpretation of transverse instability}

In order to understand the qualitative mathematical difference between the twodimensional modulational stability properties of a strictly cold plasma and those of a warm plasma (even at arbitrarily low temperature), it is instructive to discuss the physical effects of the transverse terms in (42) and (43): First, consider what happens if both components of the plasma have exactly zero temperature, implying vanishing $v_{s}$. If the envelope has some small transverse variations, then (42) shows that $\psi$ may be obtained by quadrature and will be driven nonzero. In general, taking it to vanish as $Z \rightarrow \infty$, as one moves along and to the back of the pulse, it will pick up a term linear in $Z$ and a term independent of $Z$. (Note that behind the pulse, $a=0$. Also these terms could be $Y$ dependent.) By (36) and (39), we see that the particles will acquire a transverse momentum since the transverse velocities will be nonzero. The direction of the transverse motion will depend on the sign of $\left(a^{*} a\right)_{Y}$, and thus as one moves transversely along the pulse, there will be regions where some particles will move to the right and some will move to the left, depending on this sign. These varying values and signs for the transverse velocities will cause rarefactions and compressions to occur. Furthermore, the farther one gets back behind the pulse, the stronger will be these regions of compression and rarefaction. This is the reason why the second-order density will contain a term proportional to $Z$, as shown by (41). Thus the 
pulse will leave a wake of density oscillations (drift waves) as it propagates through the medium.

Now the last term in (43) could possibly affect modulational stability or instability. However in the case of an electron-ion plasma, due to the presence of the mass terms in (42), any correction would be too small to be of consequence. For an electron-positron plasma, the correction would be of the same sign (although an integral) and therefore could not affect the modulational stability of this case.

Next consider what happens if we have a small but nonzero thermal velocity, $v_{s}$. Now (42) becomes a linear second-order hyperbolic partial differential equation with characteristics. The first change that occurs is that the growth of $\psi$ in $Z$, as one moves back and to the rear of the pulse, is limited by the transverse width of the pulse. This is simply because when one is sufficiently far behind the pulse, there will be no characteristic lines that can intersect the pulse. Thus the wake will be limited in extent. Also, as seen in Sect. 4.3, the modulational stability could now be seriously affected, particularly if there were shortwavelength transverse variations or if the group velocity were unusually small. Basically one can see this just by assuming a plane wave solution and considering only transverse variations. Now (42) can be solved for $\psi_{Y}$ by quadrature and then the nonlinear coefficient in (43) becomes

$$
\text { Nonlinear coefficient } \rightarrow C_{H}+3 C_{R}+C_{P}+\frac{e^{2} \omega_{p}^{2} v_{g}{ }^{2}}{m M v_{s}{ }^{2}\left(v_{g}{ }^{2}-v_{s}^{2}\right)},
$$

This can be a major correction, particularly if $v_{s}$ is small. It is positive if $v_{g}{ }^{2}>v_{s}{ }^{2}$. Thus for the electron-ion plasma, it increases the nonlinear coefficient and makes the modulational instability even stronger. For an electron-positron plasma, it can easily overwhelm the net negative value of the first three coefficients and drive the pulse modulationally unstable.

The source of this instability is a resonance between an acoustic wave and the propagating electromagnetic wave. Let the background pulse be a plane wave. Now let there be a small disturbance with a wavevector $K_{Y} \hat{y}+K_{Z} \hat{z}$. The acoustical part of the wave will propagate in the direction of $\vec{K}$ at the acoustical velocity of $v_{s}$ and with a frequency of $v_{s} K$. Meanwhile the electromagnetic part is a disturbance on the underlying electromagnetic oscillations. For $K_{Y}$ small in comparison to the zeroth-order wavevector $k$, the group velocity of the electromagnetic disturbance will be essentially only in the $Z$ direction and the disturbance will propagate at the group velocity of $v_{g}$ and at a frequency of $v_{g} K_{Z}$. If these frequencies ever match, then the waves can resonate. The condition for matching is

$$
v_{g}^{2} K_{Z}^{2}=v_{s}^{2}\left(K_{Y}^{2}+K_{Z}^{2}\right),
$$

and is exactly the condition for the vanishing of the left-hand side of (42). One can also understand that this is a threshold condition, since if $K_{Y}$ is less than this value, then no particles can ever move far enough transversely to resonate with the electromagnetic disturbance. On the other hand, if $K_{Y}$ exceeds this value, then there will always be some particles that are moving somewhat slower and they will be able to resonate with the wave. 
The preceeding discussion considered the limiting behavior of solutions of the 2DNLS for $v_{s}^{2} / v_{g}^{2} \rightarrow 0$. One should keep in mind however that the limiting behavior of the original physical system will depend on the relative values of $v_{s}^{2} / v_{g}^{2}$ and $\epsilon$. If in a physical situation the gas temperature is such that $v_{s}^{2} / v_{g}^{2}$ is comparable with $\epsilon$ or smaller, then $\alpha$ as given by (47f) is of this magnitude. (For example, for the parameters reported in Borisov et al. (1992a), this occurs at temperatures below about $10^{7} \mathrm{~K}$.) Strictly speaking, a special expansion procedure would be required for this case which would place the term involving $\alpha$ in Eq. (1) on the same footing with the next higher-order terms.

\section{Remarks and Outlook}

As for our results concerning modulational instability, integrability, and self-focusing of the Zakharov equations in the envelope approximation (12), further work is needed to determine the relevance for the full system (7), but we hope that the parameter regimes determined in this paper will give hints as to what can be expected in future experiments, both real and numerical.

In both of the systems studied here, the transition from one to two spatial dimensions introduces a new degree of freedom (the second slow potential) into the nonlinear dynamics, which in each case is described by the 2DNLS. Qualitatively new phenomena arise, such as self-focusing, 2D solitons, decay of "longitudinal" 1D solitons, and "transverse" modulational instability. Generally speaking, the applicability of 1D results is therefore severely limited. However, a 1D description still may retain some qualitative validity on intermediate timescales, especially if the coupling to the second potential involves a second small parameter, here $v_{s} / v_{g}$ (in addition to the "slowness" parameter, our $\epsilon$ ). The discussion of Section 4 for example implies that the timescale for breakdown of the 1D picture for EM waves in a warm, unmagnetized plasma is long, even compared to the timescale for nonlinear $1 \mathrm{D}$ effects, if $v_{s} / v_{g}<<1$. Another example is provided by decay of $1 \mathrm{D}$ solitons (Ablowitz \& Segur, 1979), which involves long wavelengths and occurs on a timescale longer than that required for formation of NLS solitons.

Considering the potential importance of self-focusing and of $2 \mathrm{D}$ solitons for applications, it would certainly be worthwhile to pursue the question of intense propagation in other $(2+1)$-dimensional plasma systems in the hope of obtaining more general operator coefficients. For example, in electromagnetic pulse propagation, if the coupling between transverse and longitudinal fluid modes is not small, this would presumably lead to shorter time scales. One place to look for stronger coupling is EM pulse propagation in a magnetized plasma, possibly warm.

\section{Acknowledgements}

This research was supported in part by the Office of Naval Research and the Air Force Office of Scientific Research. 


\section{REFERENCES}

Ablowitz, M. \& Segur, H. 1979 J. Fluid Mechanics 92, 691.

Borisov, A. et al. 1992a Phys. Rev. Lett. 65, 1753.

Borisov, A. et al. 1992b Phys. Rev. A 45, 5830.

Djordjevic, V. \& Redekopp, L. 1977 J. Fluid Mechanics 79, 703.

Goldman, M. 1984 Rev. Mod. Phys. 56, 709.

Karpman, V. 1990 "Nonlinear Interaction between Short and Long Waves," in Compte \& Boccara, "Partially Integrable Evolution Equations in Physics," 83.

Karpman, V. \& Washimi, H. 1977 J. Plasma Physics 18, 173.

Kates, R. \& Kaup, D. 1989 J. Plasma Physics 42, 507 (Paper I).

Kates, R. \& Kaup, D. 1989 J. Plasma Physics 42, 521 (Paper II).

Kates, R. \& Kaup, D. 1991 J. Plasma Physics 46, 85 (Paper III).

Kates, R. \& Kaup, D. 1992 J. Plasma Physics 48, 119 (Paper IV).

Kates, R. \& Kaup, D. 1993 J. Plasma Physics 48, 397 (Paper V).

Shukla, P. \& Stenflo, L. 1984 Phys. Rev. A30, 2110.

Spatschek, K 1977 J. Plasma Physics 18, 293.

Zakharov, V.E. 1972 Soviet Physics JETP 35, 908 (Zh. Eksp. Teor. Fiz. 62 $1745)$. 
- 